\title{
Large-N string tension from rectangular Wilson loops
}

\section{Robert Lohmayer*†}

Rutgers University, Department of Physics and Astronomy, Piscataway, NJ 08854, USA

E-mail: lohmayer@physics.rutgers.edu

\section{Herbert Neuberger}

Rutgers University, Department of Physics and Astronomy, Piscataway, NJ 08854, USA

E-mail: neuberg@physics.rutgers.edu

In pure $\mathrm{SU}(N)$ gauge theory in four dimensions, we determine the string tension at large $N$ from smeared rectangular Wilson loops on the lattice. We learn how well loops of sizes barely on the strong-coupling side of the large- $N$ transition in their eigenvalue distribution can be described by effective string theory.

The 30th International Symposium on Lattice Field Theory

June 24 - 29, 2012

Cairns, Australia

\footnotetext{
*Speaker.

${ }^{\dagger}$ Research supported in part by the DOE, grant number DE-FG02-01ER41165.

¥Research supported in part by the DOE, grant number DE-FG02-01ER41165.
} 


\section{Introduction}

We study Wilson loop operators $W(\mathscr{C})$ in four-dimensional Euclidean $\mathrm{SU}(N)$ pure gauge theory, where $\mathscr{C}$ is a rectangular curve in $\mathbb{R}^{4}$. Perimeter- and corner-divergences of $W$ are eliminated by a (continuous) smearing procedure [1,2], where the associated smearing parameter $s$ of dimension length squared introduces an effective thickness for the curve $\mathscr{C}$.

In the infinite- $N$ limit, the eigenvalue spectrum of the Wilson loop matrix exhibits a nonanalyticity [1,3], separating a weakly-coupled short-distance regime from a qualitatively different strongly-coupled long-distance regime. At the transition point, the gap around -1 in the eigenvalue spectrum just closes. While smaller loops are insensitive to the compactness of $\mathrm{SU}(N)$, the full group is explored for larger loops (a key ingredient for confinement). The transition point, which depends on the shape of $\mathscr{C}$, provides a natural scale for matching perturbation theory to the longdistance description provided by effective string theory [4]. Our goal is to determine, by numerical lattice gauge theory methods, how well loops of sizes barely on the strong-coupling side of the large- $N$ transition can be described by effective string theory.

Using the standard single-plaquette Wilson action, we have obtained Monte Carlo estimates for smeared rectangular Wilson loops on a hypercubic lattice for various $N$ 's, couplings, volumes, and loop sizes from a database of 160 uncorrelated equilibrated gauge fields. All statistical errors quoted below are determined by jackknife with the elimination of one single gauge configuration from the set of 160 at a time. The range of couplings we use is $0.359 \leq b \leq 0.369$, spaced by $\Delta b=$ 0.001 (the upper bound prevents spontaneous $Z^{4}(N)$ symmetry breaking [5] on all our volumes $\left.V \geq 12^{4}\right)$. Satisfactory statistical independence for our observables is obtained for gauge fields at neighboring $b$ 's being separated by 500 complete $\mathrm{SU}(2)$ updates combined with 500 complete over-relaxation passes. The $N \rightarrow \infty$ limit is taken at fixed $b=\frac{\beta}{2 N^{2}}$. The set of $N$-values we use consists of $N=7,11,13,19,29$. For the continuous smearing parameter, denoted by $S$ on the lattice, we mainly use $0.2 \leq S \leq 0.4$. The Wilson loops $W_{N}$ on the lattice are defined by

$$
W_{N}\left(L_{1}, L_{2}, b, S, V\right)=\frac{1}{N}\left\langle\operatorname{Tr} \prod_{l \in \mathscr{C}} U_{l}\right\rangle .
$$

The product is over the links $l$ in the order they appear when one goes once round $\mathscr{C}$, a rectangle of sides $L_{1,2}$. All our fits are applied to

$$
w_{N}\left(L_{1}, L_{2}, b, S, V\right)=-\log W_{N}\left(L_{1}, L_{2}, b, S, V\right) .
$$

When the loops are square, the two variables $L_{1}, L_{2}$ are replaced by one $L$ with the understanding that $L_{1,2}=L$.

In Sec. 2, we present our results for the large- $N$ string tension obtained exclusively from square loops. In Sec. 3, we extract a purely loop-shape dependent number from the data and compare it to the prediction made by effective string theory. For a more detailed presentation and discussion we refer to Ref. [6].

\section{String tension from square loops}

We first want to determine $\lim _{N \rightarrow \infty}\left(\lim _{V \rightarrow \infty} w_{N}(L, b, S, V)\right)$ for square $L \times L$ loops. In principle, large- $N$ reduction provides a shortcut for the infinite-volume limit. However, this requires tests and 
fits since finite-volume effects depend on $V, N, b$ and $L$. We use two different methods to compute the limit:

\section{- Method 1)}

At fixed $N$, we compute $w_{N}$ on volumes that are sufficiently large for finite-volume effects to be negligible, then we determine $w_{\infty}(V=\infty)$ (other arguments are omitted) by fitting $w_{N}(V=\infty)$ to

$$
w_{N}(V=\infty)=w_{\infty}(V=\infty)+\frac{a_{1}(V=\infty)}{N^{2}}+\frac{a_{2}(V=\infty)}{N^{4}} .
$$

We have evidence (strong for $N=7$ and $N=11$, not that strong for $N=19$ and rather weak for $N=29$ ) that volumes $V=24^{4}, 18^{4}, 14^{4}, 12^{4}$ are sufficiently large for $N=7,11,19,29$, respectively. This statement applies to the specific set of couplings and loop sizes we use.

- Method 2)

The second method makes use of large- $N$ reduction. At fixed $V$, we first take the limit $N \rightarrow \infty$ of $w_{N}(V)$ by fitting

$$
w_{N}(V)=w_{\infty}(V)+\frac{a_{1}(V)}{N^{2}}+\frac{a_{2}(V)}{N^{4}} .
$$

So long as the center symmetry stays unbroken, there is no volume dependence in the infinite$N$ theory, i.e., $w_{\infty}(V)=w_{\infty}(V=\infty)$. We determine $w_{\infty}\left(V=12^{4}\right)$ from $N=11,13,19,29$ [method 2a)] and $w_{\infty}\left(V=14^{4}\right)$ from $\left.N=7,11,13,19[\operatorname{method} 2 \mathrm{~b})\right]$.

We obtain reasonable values of $\chi^{2} / N_{\text {dof }}$ for the fits ${ }^{1}$ and good agreement (compatible with the statistical accuracy of about $0.1 \%$ ) between the three results for $\lim _{N, V \rightarrow \infty} w_{N}(V)$. Truncating the expansions (2.1) and (2.2) at $\mathscr{O}\left(N^{-2}\right)$ would result in very large $\chi^{2} / N_{\text {dof }}$, so $a_{2}$ cannot be set to zero. Including the $N=29$ result in the fit (2.2) for $V=12^{4}$ is crucial for $2 \mathrm{a}$ ) to agree with $2 \mathrm{~b}$ ) and 1) for large loops and large $b$. Including $N=7$ in the $V=12^{4}$ fit would require an additional $1 / N^{6}$ correction in (2.2). When the lattice size $V^{\frac{1}{4}}$ is getting close to the critical lattice size $L_{c}(b)$ at which the center symmetry brakes, there is no useful information to be gained about the $N, V=\infty$ limit from numbers obtained at low values of $N$. Since the required computation time scales as $N^{3} V, 2 \mathrm{a}$ ) is about 1.75 times more expensive than $2 \mathrm{~b}$ ) and 1) is about 2.5 times more expensive than $2 b$ ). However, it is hardly possible to conclude from $2 b$ ) or $2 a$ ) alone that the estimates for $w_{\infty}(V)$ are reliable. We became confident that we have correctly determined $\lim _{N, V \rightarrow \infty} w_{N}(V)$ only after having obtained agreeing results from 1), 2a) and $2 b$ ).

For fixed $b$ and $S$, we use the shorthand notation $w_{\infty}(L) \equiv \lim _{N, V \rightarrow \infty} w_{N}(L, b, S, V)$ and expect

$$
w_{\infty}(L)+\frac{1}{4} \log L^{2}=c_{1}+c_{2} L+\sigma L^{2}+\mathscr{O}\left(\frac{1}{\sigma L^{2}}\right) .
$$

The log term comes from the determinant of small fluctuations around the minimal area configuration in the effective string description. We shall return to it in Sec. 3. For now, its presence is just assumed because including it gives good fits while excluding it gives bad fits.

\footnotetext{
${ }^{1} 2$ a) is an exception where we have $\chi^{2} / N_{\text {dof }}$ up to 6 for $b \leq 0.361$. This probably reflects the impact of the infinite- $N$ bulk transition at $b=0.360$.
} 
Neglecting corrections of order $\frac{1}{\sigma L^{3}}$, we fit

$$
\frac{1}{2}\left(w_{\infty}(L+1)-w_{\infty}(L)+\frac{1}{2} \log \left(1+\frac{1}{L}\right)\right)=\sigma\left(L+\frac{1}{2}\right)+\frac{c_{2}}{2}+\mathscr{O}\left(\frac{1}{\sigma L^{3}}\right)
$$

to a straight line as a function of $L+\frac{1}{2}$ to determine the string tension $\sigma$ and the coefficient of the perimeter term $c_{2}$ (see Fig. 1 for some examples). Most $5 \times 5$ loops fall into the neighborhood of the large- $N$ phase transition in the eigenvalue spectrum of the Wilson loop matrix for the $b$ and $S$ values we work with. Physically smaller loops will have a single-eigenvalue distribution which has a gap around -1 . Therefore, we only use loop sizes in the range $6 \leq L \leq 9$ to determine the string tension.

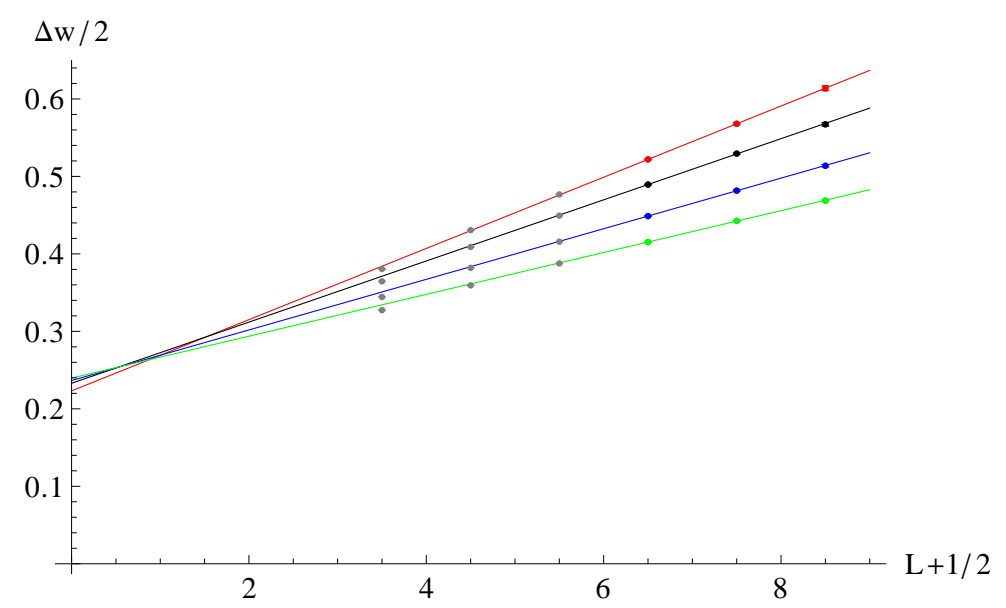

Figure 1: Plots of $\frac{\Delta w}{2}=\frac{1}{2}\left(w_{\infty}(L+1)-w_{\infty}(L)+\frac{1}{2} \log \left(1+\frac{1}{L}\right)\right)$ obtained with method 1) as a function of $L+\frac{1}{2}$ at $S=0.4$ and $b=0.36$ (red), $b=0.362$ (black), $b=0.365$ (blue) and $b=0.368$ (green). Error bars are not visible in the plot. The straight lines show linear fits through the corresponding data points. Only points $6<L+\frac{1}{2}<9$ are used in the fits.

The results obtained for $\sigma$ using the different methods for computing $w_{\infty}$ agree with each other within statistical errors, which are smallest for method 1). See Fig. 2 for a plot. As expected, $\sigma$ does not depend on the smearing level $S$ within the errors (which increase with decreasing $S$ ).

A scale length in lattice units denoted by $\xi_{c}(b)$ is used to carry out extrapolations to the continuum limit. It is defined at $N=\infty$ using a three-loop calculation of the $\beta$-function for the lattice coupling. The coefficients are written as $\bar{\beta}_{0}=\frac{\beta_{0}}{N}=\frac{11}{48 \pi^{2}}, \bar{\beta}_{1}=\frac{\beta_{1}}{N^{2}}=\frac{34}{3\left(16 \pi^{2}\right)^{2}}$, and $\bar{\beta}_{2}=\lim _{N \rightarrow \infty} \frac{\beta_{2}}{N^{3}} \approx-3.12211 \times 10^{-5}$. Integrating the RG flow, we define:

$$
\xi_{c}(b)=0.26\left(\frac{\bar{\beta}_{1}}{\bar{\beta}_{0}^{2}}+\frac{b_{I}(b)}{\bar{\beta}_{0}}\right)^{-\frac{\bar{\beta}_{1}}{2 \bar{\beta}_{0}^{2}}} \exp \left[\frac{b_{I}(b)}{2 \bar{\beta}_{0}}\right] \exp \left[\frac{\bar{\beta}_{2}}{2 \bar{\beta}_{0}^{2} b_{I}(b)}\right] .
$$

Above we have replaced the gauge coupling $b$ by $b_{I}(b)=\lim _{N, V \rightarrow \infty} b W_{N}(L=1, b, S=0, V)$, a substitution known as tadpole improvement. The definition of $\xi_{c}(b)$ is taken to match with [7]. We only added a numerical prefactor to make $\xi_{c}(b) \approx L_{c}(b)$, where $L_{c}(b)$ is given in $[5]^{2}$.

\footnotetext{
${ }^{2}$ This approximation is good to $10-15 \%$ in our range of couplings and would become exact at $b=\infty$.
} 
We separately carry out two two-parameter fits of the relation between the string tension $\sigma(b)$ and $\xi_{c}(b)$. The two pairs of parameters are denoted by $d_{0}, d_{1}$ and $f_{0}, f_{1}$ :

$$
\sigma(b)=\frac{d_{0}}{\xi_{c}(b)^{2}}+\frac{d_{1}}{\xi_{c}(b)^{4}}, \quad \text { and } \quad \frac{1}{\xi_{c}(b)^{2}}=f_{0}^{-1} \sigma(b)+f_{1} \sigma(b)^{2} .
$$

We use ranges $0.359 \leq b \leq 0.369$ (range A) and $0.362 \leq b \leq 0.367$ (range B). We also use the limited $b$ range (B) since we have observed increasing $\chi^{2}$,s for the infinite- $N, V$ extrapolations using method 2a) for $b \leq 0.361$, as mentioned above. Another reason is that finite-volume effects increase with increasing $b$. This reason only applies to method 1 ). The difference between the two fits is a simple indicator of systematic errors induced by the truncation of the perturbative series. We find that these particular systematic deviations are of the same order as the statistical errors.

Our result for the infinite- $N$ continuum string tension is given by $\lim _{b \rightarrow \infty} \sigma(b) \xi_{c}^{2}(b)=1.6(1)(3)$. The first error is statistical and the second systematic. The systematic error is more of a guess than a well founded estimate. In terms of $\Lambda_{\overline{M S}}$, this translates to $\sigma / \Lambda_{\overline{M S}}^{2}=3.4(2)(6)$.

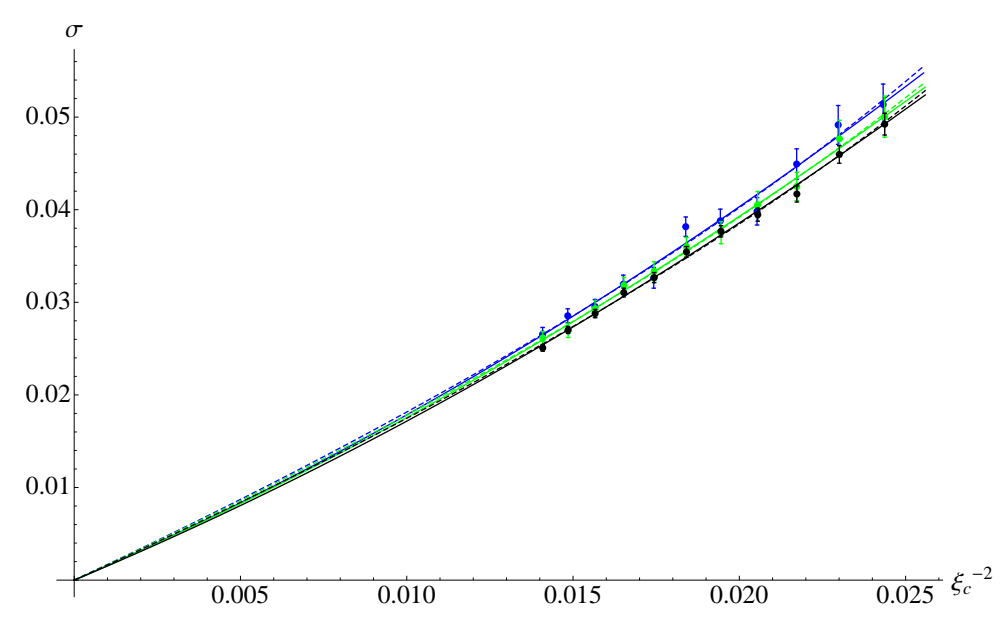

Figure 2: Plots of $\sigma$ as a function of $\xi_{c}^{-2}$ : method 1) in black, method 2a) in green, method 2b) in blue, together with corresponding fit functions (the fits are obtained using $0.359 \leq b \leq 0.369$ ).

Our central number is 2-3 standard deviations smaller than that of Allton et al. [7] $\left(\sigma / \Lambda_{\bar{M} S^{2}}=\right.$ $3.95(3)(64)$ at $N=\infty)$. Their numbers were extracted from Polyakov loops which are substantially longer than one side of our square loops. The systematic errors are dominated by the continuum extrapolation and their relative size is roughly the same for us.

A previous estimate for the string tension at infinite $N$ extracted from rectangular Wilson loops has been given in [8]. Expressed in terms of our variables it is $\left.\sigma \xi_{c}^{2}\right|_{b_{I}=0.182}=2.2(3)$. While writing up our paper [6] a new study [9] appeared which also deals with rectangular Wilson loops. These authors obtain $\sigma / \Lambda_{\bar{M} S^{2}}=3.63(3)$ (statistical error) at $N=\infty$ if they apply the continuum extrapolation method of [7]. This number is fully consistent with ours and has very small errors by comparison.

There seems to be a disagreement at the statistical level between [7] and our result which agrees with that of [9]. The result of [8] seems to side with that of [7], but has too large errors to be sure. The systematic errors are too large to claim evidence for a difference between the string 
tension extracted from Wilson loops and that extracted from Polyakov loop correlators, which would be very difficult to accept at the theoretical level.

\section{Shape dependence}

We now turn to a study of the shape dependence of the scale-independent term in $w_{N}$ and compare it with the effective-string prediction. For rectangular $L_{1} \times L_{2}$ loops it is convenient to introduce the modular invariant shape parameter

$$
\zeta=\frac{L_{1}}{L_{2}}+\frac{L_{2}}{L_{1}}
$$

The accuracy we now need does not permit taking the $N \rightarrow \infty$ limit. We therefore restrict our attention to the $N=7,11$ data. We shall see that the numbers we compute are identical within errors for $N=7$ and $N=11$, indicating that it is unlikely that they will change in a substantial manner in the $N=\infty$ limit.

At fixed $b, S, V$, and fixed finite $N$, we expect (arguments $b, S, V$ are omitted)

$$
w_{N}\left(L_{1}, L_{2}\right)+\frac{1}{4} \log L_{1} L_{2}=c_{1, N}(\zeta)+c_{2, N} \frac{L_{1}+L_{2}}{2}+\sigma_{N} L_{1} L_{2}+\mathscr{O}\left(\frac{1}{\sigma_{N} L_{1} L_{2}}\right) .
$$

After having determined the lattice string tension $\sigma_{N}$ and the coefficient of the perimeter term $c_{2, N}$ from square $L \times L$ loops (using the method described in Sec. 2) at fixed $N, b, S, V$, we fit $w_{N}(L, L)+\frac{1}{4} \log L^{2}-\sigma_{N} L^{2}-c_{2, N} L$ to a constant, $c_{1, N}(\zeta=2$ ) (using loop sizes $6 \leq L \leq 10$ ). Next, we analyze the results obtained for a sequence of rectangular loops at the same $b, S, V, N$ with $L_{2}=2 L_{1}$, i.e., $\zeta=\frac{5}{2}$ fixed. Using the results for $\sigma_{N}$ and $c_{2, N}$ obtained from square loops, we then determine $c_{1, N}\left(\zeta=\frac{5}{2}\right)$ by fitting $w_{N}(L, 2 L)+\frac{1}{4} \log \left(2 L^{2}\right)-\sigma_{N} 2 L^{2}-c_{2, N} \frac{3}{2} L$ to a constant (using $4 \leq L \leq 7)$. Figure 3 shows a plot of $c_{1, N=11}(2.5)-c_{1, N=11}(2)$ as a function of $b$. Within statistical errors, our results for $c_{1, N}(2.5)-c_{1, N}(2)$ do not depend on $b, S$, or $N$. The effective-string prediction for $c_{1}(2.5)-c_{1}(2)$ is

$$
\frac{1}{2} \log \left(\frac{\eta(2 i) \eta(i / 2)}{\eta(i)^{2}}\right) \approx-0.08664
$$

where $\eta(x)$ is the eta-function. We find that the effective-string prediction is smaller than the observed values by a factor of about 1.5 to 1.7 .

We use sequences of $L \times L \pm 1$ loops to cross check our results for the string tension and the shape dependence of $c_{1, N}$. Furthermore, we simultaneously fit $L \times L, L \times 2 L$, and $L \times L \pm 1$ loops to the functional form (3.2), where we expand $c_{1, N}$ around $\zeta=2$ and allow the coefficient of the $\log L_{1} L_{2}$ term to become a fit parameter. This analysis confirms the expected value of $1 / 4$ for the coefficient of the log term as well as the results for $\sigma_{N}$ and $c_{1, N}$ presented above.

This means that one prediction coming from the determinant of Gaussian surface fluctuations in the effective string description works close to the large- $N$ transition in the eigenvalues and the other does not. However, these two predictions are somewhat different even within effective string theory (see [6] for details).

Our result for the shape dependence of the scale-independent term is in agreement with [9] who independently report a deviation from effective string theory. 


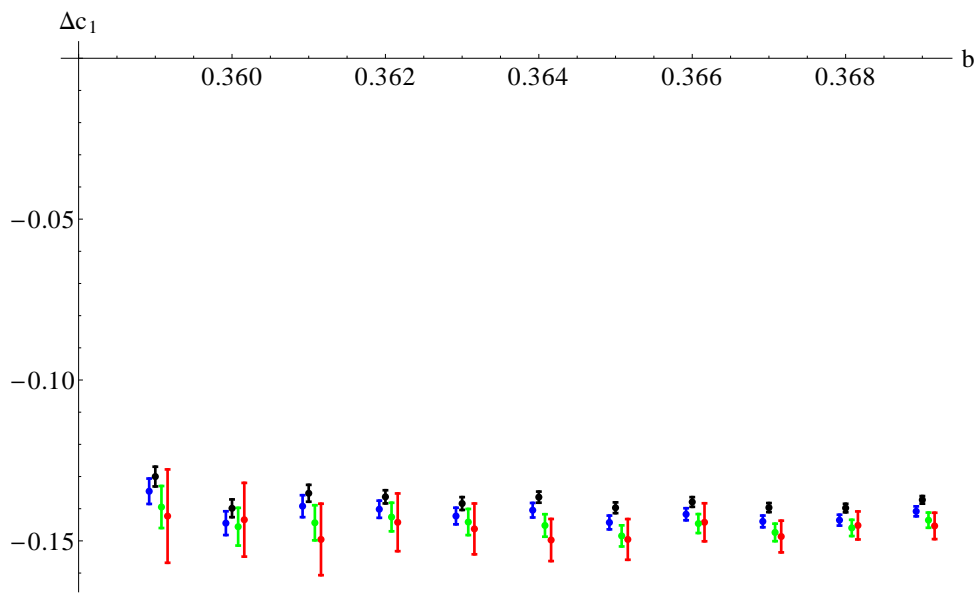

Figure 3: Plot of $c_{1, N}(2.5)-c_{1, N}(2)$ for $N=11$ (on $V=18^{4}$ ) as a function of $b$ for $S=0.2$ (red), $S=0.28$ (green) $S=0.4$ (blue), and $S=0.52$ (black).

A detailed discussion of possible explanations (from perturbation theory and by higher-order terms in the string expansion) is presented in [6]. Our data is not conclusive enough to settle this issue. We think that the shape dependence of planar Wilson loops presents an interesting case for testing the limitations of the effective string approach which deserves further study in the future.

\section{References}

[1] R. Narayanan and H. Neuberger, Infinite $N$ phase transitions in continuum Wilson loop operators, JHEP 03 (2006) 064, [hep-th/ 0601210 ].

[2] R. Lohmayer and H. Neuberger, Continuous smearing of Wilson Loops, PoS LATTICE2011 (2011) 249, [arXiv:1110.3522].

[3] R. Lohmayer and H. Neuberger, Non-analyticity in scale in the planar limit of QCD, Phys. Rev. Lett. 108 (2012) 061602, [arXiv:1109.6683].

[4] O. Aharony and M. Field, On the effective theory of long open strings, JHEP 01 (2011) 065, [arXiv:1008.2636].

[5] J. Kiskis, R. Narayanan, and H. Neuberger, Does the crossover from perturbative to nonperturbative physics in QCD become a phase transition at infinite N?, Phys. Lett. B574 (2003) 65-74, [hep-lat/0308033].

[6] R. Lohmayer and H. Neuberger, Rectangular Wilson Loops at Large N, JHEP 1208 (2012) 102, [arXiv:1206.4015].

[7] C. Allton, M. Teper, and A. Trivini, On the running of the bare coupling in $S U(N)$ lattice gauge theories, JHEP 07 (2008) 021, [arXiv: 0803 . 1092].

[8] J. Kiskis and R. Narayanan, Computation of the string tension in four-dimensional Yang-Mills theory using large N reduction, Phys. Lett. B681 (2009) 372-375, [arXiv:0908. 1451 ].

[9] A. Gonzalez-Arroyo and M. Okawa, The string tension from smeared Wilson loops at large N, arXiv:1206.0049. 